\title{
Finite-Time Simultaneous Stabilization of a Set of Nonlinear Singular Systems
}

\author{
Liying Sun $\mathbb{D D}^{1}$ and Meiqing $\mathrm{Li} \mathbb{1}^{2}$ \\ ${ }^{1}$ School of Business, Shanghai Dianji University, Shanghai 201306, China \\ ${ }^{2}$ School of Automation, Nanjing University of Science and Technology, Nanjing 210094, China \\ Correspondence should be addressed to Liying Sun; ss_sunly@163.com
}

Received 19 April 2021; Accepted 11 June 2021; Published 19 June 2021

Academic Editor: Cuimei Jiang

Copyright (c) 2021 Liying Sun and Meiqing Li. This is an open access article distributed under the Creative Commons Attribution License, which permits unrestricted use, distribution, and reproduction in any medium, provided the original work is properly cited.

\begin{abstract}
This study addresses the problems of finite-time simultaneous stabilization for two nonlinear singular systems and more than two nonlinear singular systems. First, we design a suitable output feedback controller and combine the two nonlinear singular systems to generate an augmented system by using an augmented technique. Based on a sufficient condition of the augmented system impulse-free, an important result, that is, the augmented system is finite-time stabilization is presented. Then, the finite-time stabilization problem of more than two singular systems is investigated by dividing the $N$ systems into two sets. Finally, a numerical example is provided to demonstrate the effectiveness of the proposed finite-time stabilization controller for two nonlinear singular systems.
\end{abstract}

\section{Introduction}

In recent years, the singular system has attracted extensive attention of researchers, which is also called the descriptor system, generalized state-space system, differential-algebraic system, and so on. Due to its unique properties and structure, the singular system is more complex than the normal state-space system. It needs to consider impulse-free (continue-time system) or causality (discrete-time system), regularity, and stable [1].

It is worth pointing out that the simultaneous stabilization problem of the normal state-space system is introduced in 1982. Abundant results of simultaneous stabilization have been obtained in recent years [2-6]. In [2], several simultaneous external and internal stabilization problems have been considered under appropriate adaptive low-and-high gain feedback controllers. Simultaneous stabilization of linear systems has been solved by the static output feedback in [3]. In [4], the simultaneous stabilization of the multiinput-multioutput system has been investigated to develop a stabilizing controller. A necessary and sufficient condition for the existence of simultaneously quadratically stabilizing state feedback laws is derived for a collection of single-input discrete-time nonlinear systems in [5]. In [6], simultaneous stabilization of a set of nonlinear port-controlled Hamiltonian systems is investigated by using the dissipative Hamiltonian structural properties. Subsequently, some results of simultaneous stabilization are obtained for singular systems $[7,8]$. Simultaneous stabilization problems are investigated for nonlinear singular systems with actuator saturation by using a suitable output feedback in [7] and an uncertain singular system with input saturation via using linear matrix inequalities in [8], respectively.

By the virtue of the method of simultaneous stabilization, the finite-time simultaneous stabilization problem is considered for the nonlinear singular system. Definition of finite-time stabilization is proposed for the linear system $[9,10]$ and then extended to the linear singular system [11]. Furthermore, the finite-time stabilization is investigated for the nonlinear discrete-time Hamiltonian singular system [12]. Finite-time stabilization, which is different from asymptotic stability [13-17], is used to describe that the state does not exceed a certain bound during a fixed finite-time interval. The finite-time stabilization of the nonlinear continue-time singular system and nonlinear discrete-time singular system is investigated via 
the state-undecomposed method in $[18,19]$, respectively. In [20], the stochastic finite-time $H_{\infty}$ filtering issue for a class of nonlinear continuous-time singular semi-Markov jump systems is discussed in the forms of strict LMIs. While, stability of the robust finite-time for linear singular Markovian jump systems with impulsive effects and time-varying norm-bounded disturbance is considered under the designed state feedback controller and estimation of domain of attraction in [21]. To our best knowledge, a few results are presented for finite-time simultaneous stabilization of the singular system. In [22], finitetime control and fault detection are studied simultaneously for the singular system via the average dwell time approach and using some novel integral inequalities. When it comes to the nonlinear singular system, the finite-time simultaneous stabilization of two nonlinear singular systems and more than two nonlinear singular systems is considered in this study. It is worth noticing that it is also called finite-time stability, i.e., states of the system reach the equilibrium point within a fixed time $T$ and stay at the equilibrium point when $t>T$ [23-29]. The finite-time robust stabilization problem of general nonlinear time-delay systems is studied based on the Hamiltonian function method and observer design in [26]. In [27], the finitetime stabilization is investigated for a class of singular systems by the constructed new Lyapunov functional, while the finitetime robust simultaneous stabilization and adaptive robust simultaneous stabilization have been investigated for nonlinear systems with time delay in $[28,29]$, respectively.

In this study, our goal is to discuss the simultaneous stabilization problem of two nonlinear singular systems or more than two nonlinear singular systems and propose the designed method of the output feedback controller. The study is divided into three parts. (i) Two nonlinear singular systems discussed about state bound in finite-time interval under the nonlinear function need not to satisfy the Lipschitz condition, and the suitable output feedback law is constructed. (ii) Based on the above method, finite-time simultaneous stabilization of more than two nonlinear singular systems is studied by dividing the $N$ systems into two sets. (iii) The numerical example of two nonlinear singular systems is used to illustrate the validity of the proposed finite-time stable controller.

\section{Finite-Time Simultaneous Stabilization of Two Nonlinear Singular Systems}

Consider the following two nonlinear singular systems:

$$
\begin{aligned}
& \left\{\begin{array}{l}
E_{1} \dot{x}=f_{1}(x)+g_{1}(x) u, \\
E_{1} x(0)=E_{1} x_{0}, \\
f_{1}(0)=0 \\
y=b_{1}(x) x
\end{array}\right. \\
& \left\{\begin{array}{l}
E_{2} \dot{\xi}=f_{2}(\xi)+g_{2}(\xi) u, \\
E_{2} \xi(0)=E_{2} \xi_{0} \\
f_{2}(0)=0 \\
\eta=b_{2}(\xi) \xi
\end{array}\right.
\end{aligned}
$$

where $x \in \mathbb{R}^{n_{1}}$ and $\xi \in \mathbb{R}^{n_{2}}$ are the states of the two systems, $u \in \mathbb{R}^{q}$ is the control input, $y, \eta \in \mathbb{R}^{m}$ are the outputs, $E_{i} \in \mathbb{R}^{n_{i} \times n_{i}}$ is the singular matrix, and $0<\operatorname{rank}\left(E_{i}\right)=r_{i}<n$; $b_{i}(x) \in \mathbb{R}^{m \times n_{i}}, g_{i}(.) \in \mathbb{R}^{n_{i} \times q}, f_{i}($.$) is a sufficiently smooth$ vector field with proper dimensions, $i=1,2$.

To facilitate the analysis of the two systems (1) and (2), we show the following definition and lemma.

Definition 1 (see [30]). A control law $u(x)$ is called an admissible control law, if for any initial condition $E x_{0}$, the resulted closed-loop descriptor system has no impulsive solution, and accordingly, the original system is called impulse controllable.

Lemma 1 (see [31]). If a scalar function $h(x)$ with $h(0)=$ $0\left(x \in \mathbb{R}^{n}\right)$ has continuous $n^{\text {th }}$ order partial derivatives, then $h(x)$ can be expressed as

$$
h(x)=a_{1}(x) x_{1}+\cdots+a_{n}(x) x_{n}=A(x) x,
$$

where $a_{i}(x), i=1,2, \ldots, n$ are the scalar functions.

As mentioned above, the two systems can be described as follows:

$$
\begin{aligned}
& \left\{\begin{array}{l}
E_{1} \dot{x}=A_{1}(x) x+g_{1}(x) u, \\
E_{1} x(0)=E_{1} x_{0}, \\
f_{1}(0)=0, \\
y=b_{1}(x) x,
\end{array}\right. \\
& \left\{\begin{array}{l}
E_{2} \dot{\xi}=A_{2}(\xi) \xi+g_{2}(\xi) u, \\
E_{2} \xi(0)=E_{2} \xi_{0}, \\
f_{2}(0)=0, \\
\eta=b_{2}(\xi) \xi .
\end{array}\right.
\end{aligned}
$$

We can design an output feedback controller:

$$
u=-K(y-\eta)
$$

where $K \in \mathbb{R}^{m \times m}$ is a symmetric matrix. Substituting (6) into systems (4) and (5), the systems can be given as follows:

$$
\begin{aligned}
& \left\{\begin{array}{l}
E_{1} \dot{x}=\left(A_{1}(x)-g_{1}(x) K b_{1}(x)\right) x+g_{1}(x) K b_{2}(\xi) \xi, \\
y=b_{1}(x) x,
\end{array}\right. \\
& \left\{\begin{array}{l}
E_{2} \dot{\xi}=\left(A_{2}(\xi)+g_{2}(\xi) K b_{2}(\xi)\right) \xi-g_{2}(\xi) K b_{1}(x) x, \\
\eta=b_{2}(\xi) \xi .
\end{array}\right.
\end{aligned}
$$

Combine the two closed-loop singular systems (7) and (8) into an augmented system:

$$
\left\{\begin{array}{l}
E \dot{X}=A(X) X \\
Y=B(X) X
\end{array}\right.
$$

where 


$$
\begin{aligned}
X & =\left[\begin{array}{l}
x \\
\xi
\end{array}\right], \\
Y & =\left[\begin{array}{l}
y \\
\eta
\end{array}\right], \\
E & =\left[\begin{array}{cc}
E_{1} & 0 \\
0 & E_{2}
\end{array}\right], \\
B(X) & =\left[\begin{array}{cc}
b_{1}(x) & 0 \\
0 & b_{2}(\xi)
\end{array}\right], \\
A(X) & =\left[\begin{array}{cc}
A_{1}(x)-g_{1}(x) K b_{1}(x) & g_{1}(x) K b_{2}(\xi) \\
-g_{2}(\xi) K b_{1}(x) & A_{2}(\xi)+g_{2}(\xi) K b_{2}(x)
\end{array}\right] .
\end{aligned}
$$

Lemma 2. If $\operatorname{rank}\left[\begin{array}{cc}0 & E \\ E & A(X)\end{array}\right]=n_{1}+n_{2}+r_{1}+r_{2}$, then system (9) is impulse-free.

Proof. From $E=\operatorname{diag}\left[E_{1}, E_{2}\right]$, we know $\operatorname{rank}(E)=r_{1}+r_{2}$. So, there exist two nonsingular matrices $M, N \in \mathbb{R}^{\left(n_{1}+n_{2}\right) \times\left(n_{1}+n_{2}\right)}$, such that

$$
\mathrm{MEN}=\left[\begin{array}{cc}
I_{r_{1}+r_{2}} & 0 \\
0 & 0
\end{array}\right]
$$

Denote

$$
\begin{aligned}
\bar{X} & =N^{-1} X=\left[\begin{array}{l}
X_{1} \\
X_{2}
\end{array}\right], \\
M A(X) N & =\left[\begin{array}{ll}
\widetilde{A}_{11}(X) & \widetilde{A}_{12}(X) \\
\widetilde{A}_{21}(X) & \widetilde{A}_{22}(X)
\end{array}\right]=\left[\begin{array}{ll}
A_{11}(X) & A_{12}(X) \\
A_{21}(X) & A_{22}(X)
\end{array}\right], \\
M B(X) & =\left[\begin{array}{l}
\widetilde{B}_{1}(X) \\
\widetilde{B}_{2}(X)
\end{array}\right]=\left[\begin{array}{l}
B_{1}(\bar{X}) \\
B_{2}(\bar{X})
\end{array}\right],
\end{aligned}
$$

where $\bar{X} \in \mathbb{R}^{n_{1}+n_{2}}, X_{1} \in \mathbb{R}^{r_{1}+r_{2}}$, and $X_{2} \in \mathbb{R}^{n_{1}+n_{2}-r_{1}-r_{2}}$. Then, system (9) can be expressed as follows:

$$
\left\{\begin{array}{l}
\dot{X}_{1}=A_{11}(\bar{X}) X_{1}+A_{12}(\bar{X}) X_{2}, \\
0=A_{21}(\bar{X}) X_{1}+A_{22}(\bar{X}) X_{2}, \\
Y=B_{1}(\bar{X}) X_{1}+B_{2}(\bar{X}) X_{2} .
\end{array}\right.
$$

Noticing that

$$
\begin{aligned}
n_{1}+n_{2}+r_{1}+r_{2} & =\operatorname{rank}\left[\begin{array}{cc}
0 & E \\
E & A(X)
\end{array}\right]=\operatorname{rank}\left[\begin{array}{cc}
M & \\
& M
\end{array}\right]\left[\begin{array}{cc}
0 & E \\
E & A(X)
\end{array}\right]\left[\begin{array}{cc}
N & \\
& N
\end{array}\right] \\
& =\operatorname{rank} A_{22}(\bar{X})+2 r_{1}+2 r_{2},
\end{aligned}
$$

we can obtain that $\operatorname{rank} A_{22}(\bar{X})=n_{1}+n_{2}-r_{1}-r_{2}$, which implies that the index of equivalent closed-loop singular system (9) is one at the equilibrium point 0 , that is, system (9) is impulse-free.

Next, we present the definition of the finite-time simultaneous stable.

Definition 2. The two nonlinear singular systems (4) and (5) are said to be the finite-time simultaneous stable (FTSS) with respect to $\left(c_{1}, c_{2}, T, R\right)$, with $0<c_{1}<c_{2}$ and $R=\operatorname{diag}\left\{R_{1}, R_{2}\right\}>0$, if $X^{T}(0) E^{T} R E X(0) \leq c_{1}$, such that $X^{T}(t) E^{T} R E X(t)<c_{2}, \forall t \in[0, T], \quad$ where $\quad x^{T}(0) E_{1}^{T} R_{1}$ $E_{1} x(0) \leq c_{11}, \quad \xi^{T}(0) E_{2}^{T} R_{2} E_{2} \xi(0) \leq c_{21}$, and $\quad c_{1} \geq c_{11}+c_{21}$, $c_{11}, c_{12}>0$.

Based on augmented system (9), we give the following result of the finite-time simultaneous stable of systems (4) and (5).
Theorem 1. If there exist symmetric positive definite matrices $Q \in \mathbb{R}^{\left(n_{1}+n_{2}\right) \times\left(n_{1}+n_{2}\right)}, P_{1} \in \mathbb{R}^{n_{1} \times n_{1}}$, and $P_{2} \in \mathbb{R}^{n_{2} \times n_{2}}$, such that

$$
\begin{gathered}
{\left[\begin{array}{cc}
\Omega_{1} & \Omega_{2} \\
\Omega_{2}^{T} & \Omega_{3}
\end{array}\right] \leq 0,} \\
\lambda_{\max }(Q) c_{1} e^{\alpha T}<\lambda_{\min }(Q) c_{2}, \\
\operatorname{rank}\left[\begin{array}{cc}
0 & E \\
E & A(X)
\end{array}\right]=n_{1}+n_{2}+r_{1}+r_{2} .
\end{gathered}
$$

Then, under the output feedback control law (6), the nonlinear singular systems (4) and (5) are finite-time simultaneous stable with respect to $\left(c_{1}, c_{2}, T, R\right)$, where

$$
\begin{aligned}
& \Omega_{1}=\left(A_{1}(x)-g_{1}(x) K b_{1}(x)\right)^{T} P_{1} E_{1}+E_{1}^{T} P_{1}\left(A_{1}(x)-g_{1}(x) K b_{1}(x)\right)-\alpha E_{1}^{T} P_{1} E_{1}, \\
& \Omega_{2}=\left(g_{1}(x) K b_{2}(\xi)\right)^{T} P_{1} E_{1}-E_{2}^{T} P_{2}\left(g_{2}(\xi) K b_{1}(x)\right), \\
& \Omega_{3}=\left(A_{2}(\xi)+g_{2}(\xi) K b_{2}(\xi)\right)^{T} P_{2} E_{2}+E_{2}^{T} P_{2}\left(A_{2}(\xi)+g_{2}(\xi) K b_{2}(\xi)\right)-\alpha E_{2}^{T} P_{2} E_{2},
\end{aligned}
$$


$R=\left[\begin{array}{cc}R_{1} & 0 \\ 0 & R_{2}\end{array}\right]>0, Q=\left[\begin{array}{cc}Q_{1} & 0 \\ 0 & Q_{2}\end{array}\right]>0, P_{1}=R_{1}^{(1 / 2)} Q_{1} R_{1}^{(1 / 2)}$, $P_{2}=R_{2}^{(1 / 2)} Q_{2} R_{2}^{(1 / 2)}, \alpha>0$, and $\lambda_{\max }\left(\right.$.) and $\lambda_{\min }$ (.) indicate the maximum and minimum eigenvalues of the argument, respectively.

Proof. According to Lemma 2 and condition (17), it is clear that augmented system (9) is impulse-free. Choose the Lyapunov function

$$
V(X(t))=X^{T}(t) E^{T} \operatorname{PEX}(t) \geq 0, \forall t \in[0, T],
$$

i.e., $V(X(t))=V_{1}(x(t))+V_{2}(\xi(t))=x^{T}(t) E_{1}^{T} P_{1} E_{1} x(t)+$ $\xi^{T}(t) E_{2}^{T} P_{2} E_{2} \xi(t) \geq 0$, where $P=\left[\begin{array}{cc}P_{1} & 0 \\ 0 & P_{2}\end{array}\right]=R^{(1 / 2)} Q R^{(1 / 2)}$.
Based on system (9),

$$
\begin{aligned}
& \dot{V}(X(t))-\alpha V(X(t))=X^{T}\left(A^{T}(X) P E+E^{T} P A(X)-\alpha E^{T} P E\right) X
\end{aligned}
$$

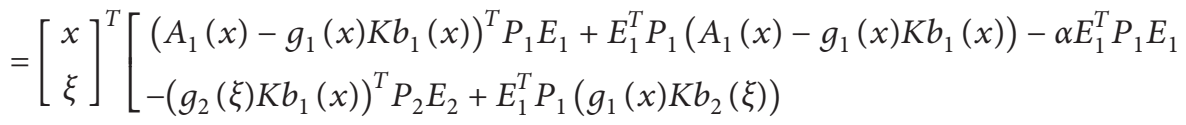

$$
\begin{aligned}
& \begin{array}{l}
\left(g_{1}(x) K b_{2}(\xi)\right)^{T} P_{1} E_{1}-E_{2}^{T} P_{2}\left(g_{2}(\xi) K b_{1}(x)\right) \\
\left(A_{2}(\xi)+g_{2}(\xi) K b_{2}(\xi)\right)^{T} P_{2} E_{2}+E_{2}^{T} P_{2}\left(A_{2}(\xi)+g_{2}(\xi) K b_{2}(\xi)\right)-\alpha E_{2}^{T} P_{2} E_{2}\left[\begin{array}{c}
x \\
\xi
\end{array}\right]
\end{array} \\
& =\left[\begin{array}{l}
x \\
\xi
\end{array}\right]^{T}\left[\begin{array}{ll}
\Omega_{1} & \Omega_{2} \\
\Omega_{2}^{T} & \Omega_{3}
\end{array}\right]\left[\begin{array}{l}
x \\
\xi
\end{array}\right] \text {. }
\end{aligned}
$$

Under condition (15), there is

$$
\dot{V}(X(t)) \leq \alpha V(X(t)), \quad \forall t \in[0, T] .
$$

$$
V(X(t)) \leq e^{\alpha t} V(X(0)), \quad \forall t \in[0, T] .
$$

Furthermore, by integrating the inequality (21) between 0 and $t$, it is clear that

$$
\begin{gathered}
V(X(t))=X^{T}(t) E^{T} \operatorname{PEX}(t)=X^{T}(t) E^{T} R^{(1 / 2)} Q R^{(1 / 2)} E X(t) \geq \lambda_{\min }(Q) X^{T}(t) E^{T} R E X(t), \\
V(X(0)) e^{\alpha t}=X^{T}(0) E^{T} P E X(0)=X^{T}(0) E^{T} R^{(1 / 2)} Q R^{(1 / 2)} E X(0) e^{\alpha t} \leq \lambda_{\max }(Q) X^{T}(0) E^{T} R E X(0) e^{\alpha T},
\end{gathered}
$$

where $\quad \lambda_{\min }(Q)=\min \left\{\lambda_{\min }\left(Q_{1}\right), \lambda_{\min }\left(Q_{2}\right)\right\}$, $\lambda_{\max }(Q)=\max \left\{\lambda_{\max }\left(Q_{1}\right), \lambda_{\max }\left(Q_{2}\right)\right\}$.

If $X^{T}(0) E^{T} R E X(0) \leq c_{1}$, taking account of (16), (22)-(24), it can be deduced that

$$
\begin{aligned}
X^{T}(t) E^{T} R E X(t) & \leq \frac{\lambda_{\max }(Q) X^{T}(0) E^{T} R E X(0)}{\lambda_{\min }(Q)} e^{\alpha T} \\
& \leq \frac{\lambda_{\max }(Q)\left(c_{1}\right)}{\lambda_{\min }(Q)} e^{\alpha T} \\
& <c_{2} .
\end{aligned}
$$

Hence, system (9) is finite-time stable with respect to $\left(c_{1}, c_{2}, T, R\right)$.

To proceed further, systems (4) and (5) can be finite-time simultaneous stabilization with respect to $\left(c_{1}, c_{2}, T, R\right)$.

and
Consider $N$ nonlinear singular systems:

$$
\left\{\begin{array}{l}
E_{i} \dot{x}^{i}=f_{i}\left(x^{i}\right)+g_{i}\left(x^{i}\right) u, \\
E_{i} x^{i}(0)=E_{i} x_{0}^{i}, \\
f_{i}(0)=0, \\
y_{i}=b_{i}\left(x^{i}\right) x^{i}, \quad i=1,2, \ldots, N,
\end{array}\right.
$$

where $x^{i} \in \mathbb{R}^{n_{i}}, y_{i} \in \mathbb{R}^{m}$, and $u \in \mathbb{R}^{q}$ are the states, the outputs, and the inputs of the $N$ systems, respectively; $g_{i}\left(x^{i}\right) \in \mathbb{R}^{n_{i} \times q}, \quad E_{i} \in \mathbb{R}^{n_{i} \times n_{i}}, 0<\operatorname{rank}\left(E_{i}\right)=r_{i}<n_{i}, \quad$ and $b_{i}(x) \in \mathbb{R}^{m \times n_{i}}, i=1,2, \ldots, N$.

Based on the method given in Section 2, we can represent system (26) into the following forms: 


$$
\left\{\begin{array}{l}
E_{i} \dot{x}^{i}=A_{i}\left(x^{i}\right) x^{i}+g_{i}\left(x^{i}\right) u \\
y_{i}=b_{i}\left(x^{i}\right) x^{i}, \quad i=1,2, \ldots, N
\end{array}\right.
$$

Suppose that $\left(i_{1}, i_{2}, \ldots, i_{N}\right)$ is an arbitrary permutation of $1,2, \ldots, N$ and that $L$ is a position integer satisfying $1 \leq L \leq N-1$. Denote $T_{1}=n_{i_{1}}+\cdots+n_{i_{L}} \quad$ and $T_{2}=n_{i_{L+1}}+\cdots+n_{i_{N}}$. We divide the $N$ systems into two sets:

$$
\begin{aligned}
& \left\{\begin{array}{l}
E_{a} \dot{X}^{a}=A_{a}\left(X^{a}\right) X^{a}+G_{a}\left(X^{a}\right) u, \\
Y_{a}=B_{a}\left(X^{a}\right) X^{a},
\end{array}\right. \\
& \left\{\begin{array}{l}
E_{b} \dot{X}^{b}=A_{b}\left(X^{b}\right) X^{b}+G_{b}\left(X^{b}\right) u, \\
Y_{b}=B_{b}\left(X^{b}\right) X^{b},
\end{array}\right.
\end{aligned}
$$

where

$$
\begin{aligned}
X^{a} & =\left[\left(x^{i_{1}}\right)^{T}, \ldots,\left(x^{i_{L}}\right)^{T}\right]^{T} \in \mathbb{R}^{T_{1}}, \\
X^{b} & =\left[\left(x^{i_{L+1}}\right)^{T}, \ldots,\left(x^{i_{N}}\right)^{T}\right]^{T} \in \mathbb{R}^{T_{2}}, \\
E_{a} & =\operatorname{diag}\left\{E_{i_{1}}, \ldots, E_{i_{L}}\right\}, \\
E_{b} & =\operatorname{diag}\left\{E_{i_{L+1}}, \ldots, E_{i_{N}}\right\}, \\
A_{a}\left(X^{a}\right) & =\operatorname{diag}\left\{A_{i_{1}}\left(x^{i_{1}}\right), \ldots, A_{i_{L}}\left(x^{i_{L}}\right)\right\}, \\
A_{b}\left(X^{b}\right) & =\operatorname{diag}\left\{A_{i_{L+1}}\left(x^{i_{L+1}}\right), \ldots, A_{i_{L}}\left(x^{i_{N}}\right)\right\}, \\
Y_{a} & =y_{i_{1}}+\cdots+y_{i_{L}}, \\
Y_{b} & =y_{i_{L+1}}+\ldots+y_{i_{N}}, \\
G_{a}\left(X^{a}\right) & =\left[g_{i_{1}}^{T}\left(x^{i_{1}}\right), \ldots, g_{i_{L}}^{T}\left(x^{i_{L}}\right)\right]^{T}, \\
G_{b}(X)^{b} & =\left[g_{i_{L+1}}^{T}\left(x^{i_{L+1}}\right), \ldots, g_{i_{N}}^{T}\left(x^{i_{N}}\right)\right]^{T}, \\
B_{a}\left(X^{a}\right) & =\left[b_{i_{1}}\left(x^{i_{1}}\right), \ldots, b_{i_{L}}\left(x^{i_{L}}\right)\right], \\
B_{b}\left(X^{b}\right) & =\left[b_{i_{L+1}}\left(x^{i_{L+1}}\right), \ldots, b_{i_{N}}\left(x^{i_{N}}\right)\right] .
\end{aligned}
$$

Design an output feedback controller:

$$
u=-K\left(Y_{a}-Y_{b}\right):=-K\left(B_{a}\left(X^{a}\right) X^{a}-B_{b}\left(X^{b}\right) X^{b}\right),
$$

where matrix $K \in \mathbb{R}^{m \times m}$ is the symmetric. Substitute (31) into systems (28) and (29), respectively. Then, the closedloop systems can be given as follows:

$$
\left\{\begin{array}{l}
E_{a} \dot{X}^{a}=\left(A_{a}\left(X^{a}\right)-G_{a}\left(X^{a}\right) K B_{a}\left(X_{a}\right)\right) X^{a}+G_{a}\left(X^{a}\right) K B_{b}\left(X^{b}\right) X^{b}, \\
Y_{a}=B_{a}\left(X^{a}\right) X^{a}
\end{array}\right.
$$

$$
\left\{\begin{array}{l}
E_{b} \dot{X}^{b}=\left(A_{b}\left(X^{b}\right)+G_{b}\left(X^{b}\right) K B_{b}\left(X_{b}\right)\right) X^{b}-G_{b}\left(X^{b}\right) K B_{a}\left(X^{a}\right) X^{a} \\
Y_{b}=B_{b}\left(X^{b}\right) X^{b}
\end{array}\right.
$$

By the system-augmentation technique, systems (32) and (33) can be transformed as

$$
\left\{\begin{array}{l}
\bar{E} \dot{\bar{X}}=\bar{A}(\bar{X}) \bar{X} \\
\bar{Y}=\bar{B}(\bar{X}) \bar{X}
\end{array}\right.
$$

where

$$
\begin{aligned}
& \bar{X}=\left[\begin{array}{c}
X^{a} \\
X^{b}
\end{array}\right], \\
& \bar{E}=\left[\begin{array}{cc}
E_{a} & 0 \\
0 & E_{b}
\end{array}\right], \\
& \bar{A}=\left[\begin{array}{cc}
A_{a}-G_{a} K B_{a} & G_{a} K B_{b} \\
-G_{b} K B_{a} & A_{b}+G_{b} K B_{b}
\end{array}\right], \\
& \bar{Y}=\left[\begin{array}{c}
Y^{a} \\
Y^{b}
\end{array}\right], \\
& \bar{B}=\left[\begin{array}{cc}
B_{a} & 0 \\
0 & B_{b}
\end{array}\right] .
\end{aligned}
$$

Based on Theorem 1, we can obtain the following result.

Theorem 2. If there exists a positive scalar $\alpha$ and symmetric positive definite matrices $\bar{Q} \in \mathbb{R}^{\left(T_{1}+T_{2}\right) \times\left(T_{1}+T_{2}\right)}$, $P_{a} \in \mathbb{R}^{T_{1} \times T_{1}}$, and $P_{b} \in \mathbb{R}^{T_{2} \times T_{2}}$, such that

$$
\left[\begin{array}{ll}
\Xi_{1} & \Xi_{2} \\
\Xi_{2}^{T} & \Xi_{3}
\end{array}\right] \leq 0
$$

$$
\lambda_{\max }(\bar{Q}) \bar{c}_{1} e^{\alpha T}<\lambda_{\min }(\bar{Q}) \bar{c}_{2}
$$

$\operatorname{Rank}\left[\begin{array}{cc}0 & \bar{E} \\ \bar{E} & \bar{A}(\bar{X})\end{array}\right]=\sum_{i=1}^{N}\left(n_{i}+r_{i}\right)$

then, under the output feedback control law (31), system (26) is finite-time simultaneous stabilization with respect to $\left(\bar{c}_{1}, \bar{c}_{2}, \bar{T}, \bar{R}\right)$, where $\bar{c}_{2}>\bar{c}_{1}>0, \bar{R}>0, K, \lambda_{\max }($.$) , and \lambda_{\min }($. are the same as those in Theorem 1. Noted that,

$$
\begin{aligned}
& \Xi_{1}=\left(A_{a}\left(X^{a}\right)-G_{a}\left(X^{a}\right) K B_{a}\left(X^{a}\right)\right)^{T} P_{a} E_{a}+E_{a}^{T} P_{a}\left(A_{a}\left(X^{a}\right)-G_{a}\left(X^{a}\right) K B_{a}\left(X^{a}\right)\right)-\alpha E_{a}^{T} P_{a} E_{a}, \\
& \Xi_{2}=\left(G_{a}\left(X^{a}\right) K B_{b}\left(X^{b}\right)\right)^{T} P_{a} E_{a}-E_{b}^{T} P_{b}\left(G_{b}\left(X^{b}\right) K B_{a}\left(X^{a}\right)\right), \\
& \Xi_{3}=\left(A_{b}\left(X^{b}\right)+G_{b}\left(X^{b}\right) K B_{b}\left(X^{b}\right)\right)^{T} P_{b} E_{b}+E_{b}^{T} P_{b}\left(A_{b}\left(X^{b}\right)+G_{b}\left(X^{b}\right) K B_{b}\left(X^{b}\right)\right)-\alpha E_{b}^{T} P_{b} E_{b} .
\end{aligned}
$$


Proof. Taking the same as Theorem 1, system (34) is impulse-free depending on Lemma 2 and condition (38). We choose a Lyapunov function $V(\bar{X}(t))=\bar{X}^{T}(t)$ $\bar{E}^{T} \bar{P} \bar{E} \bar{X}(t) \geq 0, \forall t \in[0, T], \quad$ where $\quad \bar{P}=\left[\begin{array}{cc}P_{a} & 0 \\ 0 & P_{b}\end{array}\right]=$
$\left[\begin{array}{cc}R_{a}^{(1 / 2)} & 0 \\ 0 & R_{\underline{b}}^{(1 / 2)}\end{array}\right]\left[\begin{array}{cc}Q_{a} & 0 \\ 0 & Q_{b}\end{array}\right]\left[\begin{array}{cc}R_{a}^{(1 / 2)} & 0 \\ 0 & R_{b}^{(1 / 2)}\end{array}\right]=\bar{R}^{(1 / 2)} \quad \bar{Q}^{(1 / 2)}$, and $\bar{Q}, \bar{P}, \bar{R}$ are the symmetric positive definite matrices.

Based on conditions (36) and (37), we have

$$
\begin{aligned}
& \dot{V}(\bar{X})-\alpha V(\bar{X})=\bar{X}^{T}\left(\bar{E}^{T} \bar{P} \bar{A}+\bar{A}^{T} \bar{P} \bar{E}-\alpha \bar{E} \bar{P} \bar{E}\right) \bar{X} \\
& =\left[\begin{array}{c}
X^{a} \\
X^{b}
\end{array}\right]^{T}\left[\begin{array}{c}
\left(A_{a}\left(X^{a}\right)-G_{a}\left(X^{a}\right) K B_{a}\left(X^{a}\right)\right)^{T} P_{a} E_{a}+E_{a}^{T} P_{a}\left(A_{a}\left(X^{a}\right)-G_{a}\left(X^{a}\right) K B_{a}\left(X^{a}\right)\right)-\alpha E_{a}^{T} P_{a} E_{a} \\
E_{a}^{T} P_{a} G_{a}\left(X^{a}\right) K B_{b}\left(X^{b}\right)-\left(G_{b}\left(X^{b}\right) K B_{a}\left(X^{a}\right)\right)^{T} P_{b} E_{b}
\end{array}\right. \\
& \left.\begin{array}{c}
\left(G_{a}\left(X^{a}\right) K B_{b}\left(X^{b}\right)\right)^{T} P_{a} E_{a}-E_{b}^{T} P_{b}\left(G_{b}\left(X^{b}\right) K B_{a}\left(X^{a}\right)\right) \\
\left(A_{b}\left(X^{b}\right)+G_{b}\left(X^{b}\right) K B_{b}\left(X^{b}\right)\right)^{T} P_{b} E_{b}+E_{b}^{T} P_{b}\left(A_{b}\left(X^{b}\right)+G_{b}\left(X^{b}\right) K B_{b}\left(X^{b}\right)\right)-\alpha E_{b}^{T} P_{b} E_{b}
\end{array}\right]\left[\begin{array}{c}
X^{a} \\
X^{b}
\end{array}\right] \\
& =\left[\begin{array}{l}
X^{a} \\
X^{b}
\end{array}\right]^{T}\left[\begin{array}{ll}
\Xi_{1} & \Xi_{2} \\
\Xi_{2}^{T} & \Xi_{3}
\end{array}\right]\left[\begin{array}{l}
X^{a} \\
X^{b}
\end{array}\right] \leq 0 \text {. }
\end{aligned}
$$

To sum up,

$$
\dot{V}(\bar{X}(t)) \leq \alpha V(\bar{X}(t)) .
$$

By integrating inequality (41) between 0 and $T$ with $t \in[0, T]$, the following result can be presented:

$$
V(\bar{X}(t)) \leq e^{\alpha t} V(\bar{X}(0)) .
$$

Next, we will prove the finite-time simultaneous stabilization for more than two nonlinear singular systems.

It is straightforward to see

$$
\begin{gathered}
V(\bar{X}(t))=\bar{X}^{T}(t) \bar{E}^{T} \bar{P} \bar{E} \bar{X}(t)=\bar{X}^{T}(t) \bar{E}^{T} \bar{R}^{(1 / 2)} \overline{Q R}^{(1 / 2)} \bar{E} \bar{X}(t) \geq \lambda_{\min }(\bar{Q}) \bar{X}^{T}(t) \bar{E}^{T} \bar{R} \bar{E} \bar{X}(t), \\
V(\bar{X}(0)) e^{\alpha t}=\bar{X}^{T}(0) \bar{E}^{T} \bar{P} \bar{E} \bar{X}(0) e^{\alpha t}=\bar{X}^{T}(0) \bar{E}^{T} \bar{R}^{(1 / 2)} \overline{Q R}^{(1 / 2)} \bar{E} \bar{X}(0) e^{\alpha t} \leq \lambda_{\max }(\bar{Q}) \bar{X}^{T}(0) \bar{E}^{T} \bar{R} \bar{E} \bar{X}(0) e^{\alpha T},
\end{gathered}
$$

where $\quad \lambda_{\max }(\bar{Q})=\max \left\{\lambda_{\max }\left(Q_{a}\right), \lambda_{\max }\left(Q_{b}\right)\right\}, \quad$ and $\lambda_{\text {min }}(\bar{Q})=\min \left\{\lambda_{\min }\left(Q_{a}\right), \lambda_{\min }\left(Q_{b}\right)\right\}$. According to the concept of finite-time simultaneous stable, from $\bar{X}^{T}(0) \bar{E}^{T} \bar{R} \bar{E} \bar{X}(0) \leq \bar{c}_{1}$ and (37), we can obtain

$$
\begin{aligned}
\bar{X}^{T}(t) \bar{E}^{T} \bar{R} \bar{E} \bar{X}(t) & \leq \frac{\lambda_{\max }(\bar{Q}) \bar{X}^{T}(0) \bar{E}^{T} \bar{R} \bar{E} \bar{X}(0)}{\lambda_{\min }(\bar{Q})} e^{\alpha T} \\
& \leq \frac{\lambda_{\max }(\bar{Q}) \bar{c}_{1}}{\lambda_{\min }(\bar{Q})} e^{\alpha T}<\bar{c}_{2} .
\end{aligned}
$$

By using the similar method as that in Theorem 1 , system (34) is finite-time stable with respect to $\left(\bar{c}_{1}, \bar{c}_{2}, \bar{T}, \bar{R}\right)$, and it can be shown that system (26) is finite-time simultaneous stabilization with respect to $\left(\bar{c}_{1}, \bar{c}_{2}, \bar{T}, \bar{R}\right)$.

\section{Illustrative Example}

In this section, we present an example to illustrate the effectiveness of the proposed method which addresses the finite-time simultaneous stabilization problem of the two nonlinear singular systems.

Example 1. Consider the two nonlinear singular systems:

$$
\begin{aligned}
& \left\{\begin{array}{l}
E_{1} \dot{x}=f_{1}(x)+g_{1}(x) u, \\
E_{1} x(0)=E_{1} x_{0} \\
f_{1}(0)=0 \\
y=b_{1}(x) x
\end{array}\right. \\
& \left\{\begin{array}{l}
E_{2} \dot{\xi}=f_{2}(\xi)+g_{2}(\xi) u \\
E_{2} \xi(0)=E_{2} \xi_{0} \\
f_{2}(0)=0 \\
\eta=b_{2}(\xi) \xi
\end{array}\right.
\end{aligned}
$$

where $x=\left[x_{1}, x_{2}\right]^{T} \in \mathbb{R}^{2}, \xi=\left[\xi_{1}, \xi_{2}\right]^{T} \in \mathbb{R}^{2}, u \in \mathbb{R}^{2}$, we give the following parameters: 


$$
\begin{aligned}
E_{1} & =\left[\begin{array}{ll}
1 & 2 \\
1 & 2
\end{array}\right], \\
E_{2} & =\left[\begin{array}{ll}
1 & 1 \\
0 & 0
\end{array}\right], \\
f_{1}(x) & =\left[\begin{array}{c}
-2 x_{1} x_{2}^{2}-4 x_{1}-5 x_{2} \\
-5 x_{1}-2 x_{2}^{3}-1.5 x_{2}
\end{array}\right], \\
f_{2}(\xi) & =\left[\begin{array}{cc}
-0.55 \xi_{1} \xi_{2}^{2}-2.3 \xi_{1}+5 \xi_{2} \\
5 \xi_{1}-2.2 \xi_{2}^{3}-2 \xi_{2}
\end{array}\right], \\
g_{1}(x) & =\left[\begin{array}{cc}
-1 & 0 \\
0 & -1
\end{array}\right], \\
g_{2}(\xi) & =\left[\begin{array}{cc}
-1 & 0 \\
0 & -1
\end{array}\right], \\
b_{1}(x) & =\left[\begin{array}{cc}
1 & 0 \\
0 & 1
\end{array}\right], \\
b_{2}(\xi) & =\left[\begin{array}{ll}
1 & 0 \\
0 & 1
\end{array}\right] .
\end{aligned}
$$

Based on Lemma 1, it can deduce that

$$
\begin{aligned}
& A_{1}(x)=\left[\begin{array}{cc}
-2 x_{2}^{2}-4 & -5 \\
-5 & -2 x_{2}^{2}-1.5
\end{array}\right], \\
& A_{2}(\xi)=\left[\begin{array}{cc}
-0.55 \xi_{2}^{2}-2.3 & 5 \\
5 & -2.2 \xi_{2}^{2}-2
\end{array}\right] .
\end{aligned}
$$

Design an output feedback controller

$$
\begin{aligned}
u & =-K(y-\eta)=-\left[\begin{array}{cc}
3 & 5 \\
5 & 0.5
\end{array}\right]\left[\begin{array}{c}
x_{1}-\xi_{1} \\
x_{2}-\xi_{2}
\end{array}\right] \\
& =\left[\begin{array}{c}
-3 x_{1}-5 x_{2}+3 \xi_{1}+5 \xi_{2} \\
-5 x_{1}-0.5 x_{2}+5 \xi_{1}+0.5 \xi_{2}
\end{array}\right] .
\end{aligned}
$$

Substituting (49) into systems (45) and (46), respectively, we obtain

$$
\begin{aligned}
& \left\{\begin{array}{l}
E_{1} \dot{x}=\left(A_{1}(x)-g_{1}(x) K b_{1}(x)\right) x+g_{1}(x) K b_{2}(\xi), \\
y=b_{1}(x) x,
\end{array}\right. \\
& \left\{\begin{array}{l}
E_{2} \dot{\xi}=\left(A_{2}(\xi)+g_{2}(\xi) K b_{2}(\xi)\right) \xi-g_{2}(\xi) K b_{1}(x), \\
\eta=b_{2}(\xi) \xi .
\end{array}\right.
\end{aligned}
$$

\footnotetext{
Based on form (9), we have
}

$$
\left\{\begin{array}{l}
E \dot{X}=A(X) X \\
Y=B(X) X
\end{array}\right.
$$

where

$$
\begin{aligned}
& X=[x \xi]^{T}=\left[\begin{array}{c}
x_{1} \\
x_{2} \\
\xi_{1} \\
\xi_{2}
\end{array}\right], \\
& E=\left[\begin{array}{llll}
1 & 2 & 0 & 0 \\
1 & 2 & 0 & 0 \\
0 & 0 & 1 & 1 \\
0 & 0 & 0 & 0
\end{array}\right] \\
& B=\left[\begin{array}{llll}
1 & 0 & 0 & 0 \\
0 & 1 & 0 & 0 \\
0 & 0 & 1 & 0 \\
0 & 0 & 0 & 1
\end{array}\right] \\
& A(X)=\left[\begin{array}{cccc}
-2 x_{2}^{2}-1 & 0 & -3 & -5 \\
0 & -2 x_{2}^{2}-1 & -5 & -0.5 \\
3 & 5 & -0.55 \xi_{2}^{2}-5.3 & 0 \\
5 & 0.5 & 0 & -2.2 \xi_{2}^{2}-2.5
\end{array}\right] \text {. }
\end{aligned}
$$

Choose

$$
\begin{aligned}
& M=\left[\begin{array}{cccc}
1 & 0 & 0 & 0 \\
0 & 0 & 1 & 0 \\
-1 & 1 & 0 & 0 \\
0 & 0 & 0 & 1
\end{array}\right], \\
& N=\left[\begin{array}{cccc}
1 & 0 & 0 & -2 \\
0 & 0 & 0 & 1 \\
0 & 0 & 1 & 0 \\
0 & 1 & -1 & 0
\end{array}\right] .
\end{aligned}
$$

According to Lemma 2, we can easy check that the augmented system is impulse-free.

Next, we give Lyapunov function $V(X(t))=X^{T}(t) E^{T} P E X(t)$, where $P=\left[\begin{array}{llll}1 & 0 & 0 & 0 \\ 0 & 2 & 0 & 0 \\ 0 & 0 & 2 & 0 \\ 0 & 0 & 0 & 4\end{array}\right]$. Let 


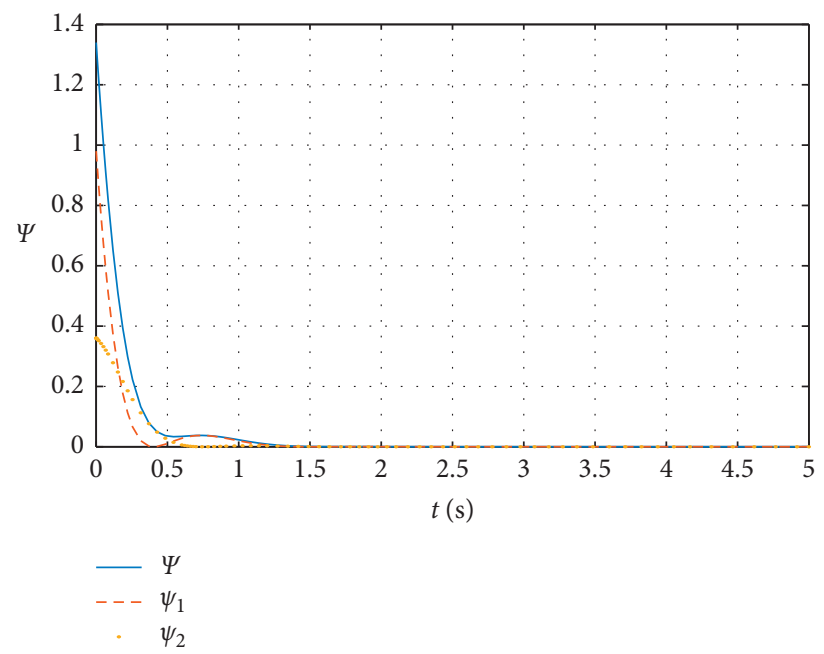

Figure 1: Responses of the values $\psi, \psi_{1}$, and $\psi_{2}$.

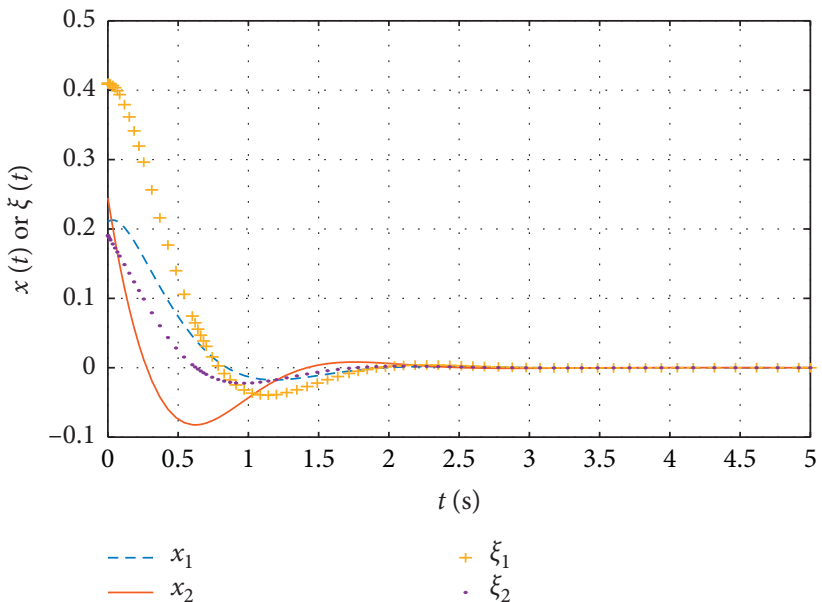

Figure 2: Responses of the state $x$ and $\xi$.

$\alpha=0.2, Q=P, R=I, c_{1}=1.34, c_{2}=8.75$, and $T=2$. By the

virtue of conditions (15) and (16) in Theorem 1, we have

$$
\begin{aligned}
& {\left[\begin{array}{ll}
\Omega_{1} & \Omega_{2} \\
\Omega_{2}^{T} & \Omega_{3}
\end{array}\right]=\left[\begin{array}{cccc}
-4 x_{2}^{2}-2.6 & -8 x_{2}^{2}-5.2 & -7 & -16 \\
-8 x_{2}^{2}-5.2 & -16 x_{2}^{2}-10.4 & 0 & -2 \\
-7 & 0 & -2.2 \xi_{2}^{2}-21.2 & -1.1 \xi_{2}^{2}-10.6 \\
-16 & -2 & -1.1 \xi_{2}^{2}-10.6 & -0.4
\end{array}\right] \leq 0,} \\
& \frac{\lambda_{\text {max }}(Q) c_{1} e^{\alpha T}}{\lambda_{\min }(Q)}=\frac{4 c_{1}}{1} e^{0.2 T}<c_{2} .
\end{aligned}
$$

Choose the initial state $E_{1} x(0)=[0.7,0.7]^{T}$ and $E_{2} \xi(0)=[0.6,0]^{T}$, then $X(0)^{T} E^{T} R E X(0) \leq c_{1}$, the trajectory of $\Psi=X(t)^{T} E^{T} R E X(t)<c_{2}, \forall t \in[0,2]$. Denote $\psi_{1}=$
$x(t)^{T} E_{1}^{T} R_{1} E_{1} x(t)$ and $\psi_{2}=\xi(t)^{T} E_{2}^{T} R_{2} E_{2} \xi(t)$. The trajectories of $\Psi, \psi_{1}$ and $\psi_{2}$, state response, and control signal are shown in Figures 1-3, respectively. Obviously, the 


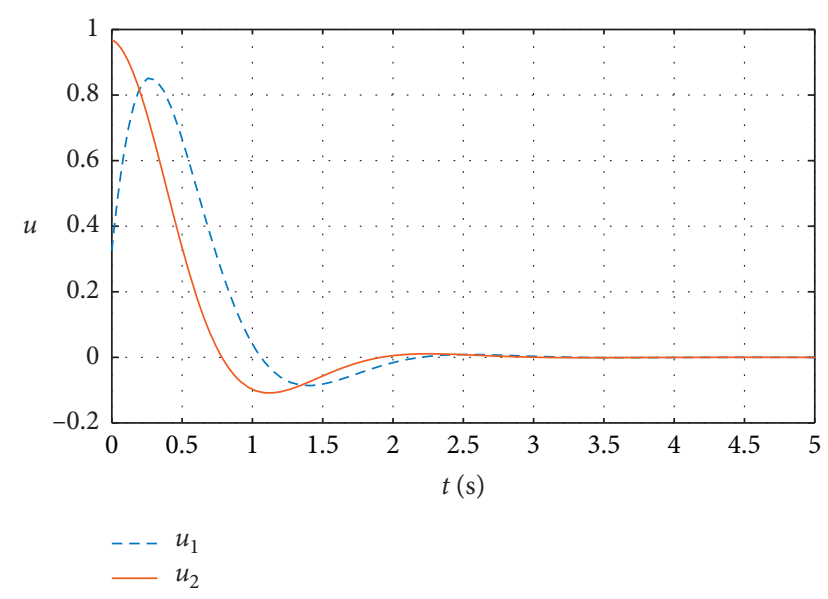

FIgURE 3: Responses of the control signal $u$.

augmented nonlinear singular system (52) is finite-time stable with respect to $(1.34,8.75,2, I)$. Hence, under the output feedback controller (49), systems (45) and (46) are finite-time simultaneous stabilization.

\section{Conclusion}

The finite-time simultaneous stabilization problem is considered for the two nonlinear singular systems or more than two nonlinear singular systems in this study. Under a suitable output feedback controller, the augmented singular system is first proved to be impulse-free and then is proved to be finite-time simultaneous stable by using the systemaugmented technique. Finally, the finite-time simultaneous stabilization problem of more than two nonlinear singular systems is investigated with the same method. Moreover, the proposed method in this study can be extended to handle finite-time $H_{\infty}$ simultaneous stabilization of the two or more than two nonlinear singular systems.

\section{Data Availability}

The data used to support the findings of this study are available from the corresponding author upon request.

\section{Conflicts of Interest}

The authors declare that they have no conflicts of interest.

\section{References}

[1] L. Dai, Singular Control Systems, Springer-Verlag, Berlin, Germany, 1989.

[2] X. Wang, A. Saberi, A. A. Stoorvogel, and P. Sannuti, "Simultaneous global external and internal stabilization of linear time-invariant discrete-time systems subject to actuator saturation," Automatica, vol. 48, no. 5, pp. 699-711, 2012.

[3] S. Kim, Y. Moon, and S. Kwon, "Simultaneous stabilization by static output feedback: a rank-constrained LMI approach," in Proceedings of the IEEE Conference on Decision and Control, pp. 5162-5167, San Diego, CA, USA, December 2006.
[4] H.-B. Chen, J. H. Chow, M. A. Kale, and K. D. Minto, "Simultaneous stabilization using stable system inversion," Automatica, vol. 31, no. 4, pp. 531-542, 1995.

[5] J. Jenq-Lang Wu, "Simultaneous quadratic stabilization for discrete-time nonlinear systems," IEEE Transactions on Automatic Control, vol. 55, no. 6, pp. 1443-1448, 2010.

[6] Y. Wang, G. Feng, and D. Cheng, "Simultaneous stabilization of a set of nonlinear port-controlled Hamiltonian systems," Automatica, vol. 43, no. 3, pp. 403-415, 2007.

[7] L. Sun, "Parallel simultaneous stabilization of a class of nonlinear descriptor systems with actuator saturation," in Proceedings of the 34th Chinese Control Conference, pp. 856-860, Hangzhou, China, July 2015.

[8] Y. Chen, T. Xu, C. Zeng, Z. Zhou, and Q. Zhang, "Simultaneous stabilization for uncertain descriptor systems with input saturation," International Journal of Robust and Nonlinear Control, vol. 22, no. 17, pp. 1938-1951, 2012.

[9] F. Amato, M. Ariola, and P. Dorato, "Finite-time control of linear systems subject to parametric uncertainties and disturbances," Automatica, vol. 37, no. 9, pp. 1459-1463, 2001.

[10] F. Amato, M. Ariola, and C. Cosentino, "Finite-time stabilization via dynamic output feedback," Automatica, vol. 42, no. 2, pp. 337-342, 2006.

[11] J. Feng, Z. Wu, J. Sun et al., " $5^{\text {th }}$ Finite time control of linear singular systems subject to parametric uncertain and disturbances," in Proceedings of the World Congress on Intelligence Control and Automation, vol. 2, no. 2, pp. 1002-1006, Hangzhou, China, October 2004.

[12] X. Lu, X. Zhang, and L. Sun, "Finite-time $\mathrm{H} \infty$ control for nonlinear discrete Hamiltonian descriptor systems," Journal of the Franklin Institute, vol. 354, no. 14, pp. 6138-6151, 2017.

[13] R. Yang and Y. Wang, "Stability for a class of nonlinear timedelay systems via Hamiltonian functional method," Science China Information Sciences, vol. 55, no. 5, pp. 1218-1228, 2012.

[14] X. Yi, R. Guo, and Y. Qi, "Stabilization of chaotic systems with both uncertainty and disturbance by the UDE-based control method," IEEE Access, vol. 8, no. 1, pp. 62471-62477, 2020.

[15] L. Liu, B. Li, and R. Guo, "Consensus control for networked manipulators with switched parameters and topologies," IEEE Access, vol. 9, pp. 9209-9217, 2021.

[16] T. Hou, Y. Liu, and F. Deng, "Stability for discrete-time uncertain systems with infinite Markov jump and time-delay," Science China: Information Sciences, vol. 64, p. 1C11, 2021.

[17] R. Peng, C. Jiang, and R. Guo, "Stabilization of a class of fractional order systems with both uncertainty and disturbance," IEEE Access, vol. 9, pp. 42697-42706, 2021.

[18] M. Li, L. Sun, and R. Yang, "Finite-time Ho control for a class of discrete-time nonlinear singular systems," Journal of the Franklin Institute, vol. 355, no. 13, pp. 5384-5393, 2018.

[19] M. Li and L. Sun, "Finite-time stabilisation for a class of nonlinear descriptor systems," IET Control Theory and Applications, vol. 12, no. 17, pp. 2399-2406, 2018.

[20] J. Wang, S. Ma, and C. Zhang, "Finite-time $\mathrm{H} \infty$ filtering for nonlinear continuous-time singular semi-markov jump systems," Asian Journal of Control, vol. 21, no. 2, pp. 1017-1027, 2019.

[21] X. Su and X. Zhao, "Robust finite-time control of descriptor Markovian jump systems with impulsive," Advances in Difference Equations, vol. 2019, no. 1, Article ID 196, 2019.

[22] M. Luo, S. Zhong, and J. Cheng, "Simultaneous finite-time control and fault detection for singular markovian jump delay systems with average dwell time constraint," Circuits, Systems, and Signal Processing, vol. 37, no. 12, pp. 5279-5310, 2018. 
[23] R. Yang and R. Guo, "Adaptive finite-time robust control of nonlinear delay Hamiltonian systems via Lyapunov-Krasovskii method," Asian Journal of Control, vol. 20, no. 1, pp. 332-342, 2018.

[24] R. Yang and L. Sun, "Finite-time robust control of a class of nonlinear time-delay systems via Lyapunov functional method," Journal of the Franklin Institute, vol. 356, no. 3, pp. 1155-1176, 2019.

[25] R. Yang, F. Zang, L. Sun, P. Zhou, and B. Zhang, "Finite-time adaptive robust control of nonlinear time-delay uncertain systems with disturbance," International Journal of Robust and Nonlinear Control, vol. 29, no. 4, pp. 919-934, 2019.

[26] R. Yang, G. Zhang, and L. Sun, "Observer-based finite-time robust control of nonlinear time-delay systems via Hamiltonian function method," International Journal of Control, vol. 4, pp. 1-18, 2020.

[27] R. Yang, L. Sun, G. Zhang, and Q. Zhang, "Finite-time stability and stabilization of nonlinear singular time-delay systems via Hamiltonian method," Journal of the Franklin Institute, vol. 356, no. 12, pp. 5961-5992, 2019.

[28] R. Yang, G. Zhang, and L. Sun, "Finite-time robust simultaneous stabilization of a set of nonlinear time-delay systems," International Journal of Robust and Nonlinear Control, vol. 30, no. 5, pp. 1733-1753, 2020.

[29] R. Yang, W. Pei, Y. Han, and L. Sun, "Finite-time adaptive robust simultaneous stabilization of nonlinear delay systems by the Hamiltonian function method," Science China Information Sciences, vol. 64, no. 6, Article ID 169201, 2021.

[30] H. Xu and K. Mizukami, "Hamilton-Jacobi equation for descriptor systems," Systems and Control Letters, vol. 21, no. 4, pp. 321-327, 1993.

[31] W. Langson and A. Allcync, "Infinite horizon optimal control of a class of nonlinear systems," in Proceedings of the 1997 American Control Conference, vol. 5, no. 1, pp. 3017-3022, Albuquerque, New Mexico, June 1997. 